\title{
On Channel Adaptive Multiple Access Control with Queued Transmission Requests for Wireless ATM
}

Vincent K. N. Lau and Yu-Kwong Kwok

Department of Electrical and Electronic Engineering

The University of Hong Kong, Pokfulam Road, Hong Kong

\begin{abstract}
In this paper, we propose a new multiple access control (MAC) protocol for wireless ATM systems, in which user demands are highly heterogeneous and can be classified as CBR, VBR, and ABR. Our protocol is motivated by two of the most significant drawbacks of existing protocols: (1) channel condition is ignored or not exploited, and (2) inflexible or biased time slots allocation algorithms are used. Indeed, existing protocols mostly ignore the burst errors due to fading and shadowing, which are inevitable in a mobile and wireless communication environment. A few protocols take into account the burst errors but just "handle" the errors in a passive manner. On the other hand, most of the existing protocols employ an inflexible or biased allocation algorithm such that over-provisioning may occur for a certain class of users at the expense of the poor service quality received by other users. Our proposed protocol, called SCAMA (synergistic channel adaptive multiple access), does not have these two drawbacks. The proposed protocol works closely with the underlying physical layer in that through observing the channel state information (CSI) of each mobile user, the MAC protocol first segregates a set of users with good CSI from requests gathered in the request contention phase of an uplink frame. The MAC protocol then judiciously allocates information time slots to the users according to their traffic types, CSI, urgency, and throughput, which are collectively represented by a novel and flexible priority function. Extensive simulations have been conducted to evaluate the SCAMA protocol.
\end{abstract}

\section{Introduction}

Wireless ATM [10], a natural extension of the wired broadband communication infrastructure (e.g., B-ISDN), is aimed at providing seamless accommodation of tetherless mobile users with QoSbased networked multimedia data services such as voice telephony, video-conferencing, or web access. However, realization of a fully functional wireless ATM system involves tackling a number intricate tasks not encountered in the fixed-wire environment. Notorious examples include multiple access control (MAC), location management, routing, etc [10]. Among these chores, MAC is one of the most important problems because the service quality and capacity of the system critically depend on the effectiveness of a MAC protocol. A MAC protocol for wireless ATM distinguishes itself from other MAC protocols in that various mechanisms are required to handle the diverse traffic demands of different services such as constant bit rate (CBR), variable bit rate (VBR), and available bit rate (ABR). CBR traffic such as voice telephony, VBR traffic such as videoconferencing, and ABR traffic such as file data have very different service requirements in terms of delay and loss tolerance, and throughput. Multiplexing these diverse services harmoniously such that reasonable QoS is maintained while at the same time maximizing the utilization of the precious channel bandwidth is a daunting and challenging task. Thus, while traditional ALOHA-type MAC protocols can handle homogeneous traffic efficiently, different techniques are needed for a wireless ATM system. As such, there are many efficient MAC protocols reported in the literature that are specially designed for wireless ATM. We describe a couple of examples below.

The DQRUMA (distributed queueing request update multiple access) protocol proposed by Karol. Liu, and Eng [4] is a well known MAC protocol for wireless ATM. In simple terms, being a FDD (frequency division duplex) protocol, DQRUMA works by dividing the uplink frame into two sections with the first section for requests contention, whereas the second section for information data transmissions. A nice and novel feature of the DQRUMA protocol is the use of a piggyback reservation field, with which a VBR user can update the traffic slot demand without the need of an extra contention. Another recently proposed MAC protocol is the DTDMA/PR (dynamic TDMA with piggybacked reservation) protocol proposed by Qiu, Li, and Ju [9]. Similar to DQRUMA, DTDMA/PR also allows VBR users to update time slots requirements using a piggyback field in an uplink information slot. A distinctive feature of DTDMA/PR is that the information slots are classified as long-term and short-term reservable. Specifically, long-term slots are for CBR and VBR users, while short-term ones are for ABR users. There are several other protocols, similar to DQRUMA and DTDMA/PR, equipped with special mechanisms for supporting heterogeneous and time varying traffic demands. The reader is referred to [11] for a detailed survey. 
Despite that these recent MAC protocols are shown to be able to handle the diverse service requirements, they still have a number of common drawbacks. Firstly, while sophisticated slot assignment strategies with articulated frame structures are proposed in these methods, none of them considers the effect of burst channel errors on protocol performance, let alone the investigation of exploiting the error characteristics to enhance performance. Essentially, these previous protocols are designed and analyzed based on the assumption that data transmission through the wireless channel is error-free. However, because the geographically scattered mobile users inevitably suffer from different degrees of fading and shadowing effects, indeed a common drawback of previous MAC protocols is that they assume the underlying physical layer always delivers a constant throughput, and as such, they may not be able to effectively utilize the precious bandwidth when the channel condition is swiftly varying among different users. Secondly, the slot allocation methods are quite inflexible in that they are designed to optimize the performance of VBR and/ or CBR users only. This lack of fairness control may lead to starvation of ABR users. Furthermore, overprovisioning becomes inevitable in these protocols. Thirdly, an important QoS metric, namely the delay jitter, is usually ignored and the protocol performance in this aspect is unknown. In view of these drawbacks, in this paper we propose a new FDD-based MAC protocol for wireless ATM with the following distinctive features:

- The proposed MAC protocol works closely with the underlying physical layer in that through observing the channel state information (CSI) of each mobile user, the MAC protocol first segregates a set of users with good CSI from requests gathered in the request contention phase of an uplink frame. The MAC protocol then judiciously allocates information time slots to the users according to their traffic types, CSI, urgency, and throughput, which are collectively represented by a novel priority function.

- The priority function used in our MAC protocol is designed to be very flexible to dynamically adjust the relative priorities of different users, both within and between traffic classes.

- Furthermore, our protocol can also minimize the delay jitter of user transmissions.

Our proposed protocol, called SCAMA (synergistic channel adaptive multiple access), also has a request queue which stores the previous requests that survive the contention but are not allocated information slots. This is to reduce the unnecessary contention loading during the contention phase. The SCAMA protocol has been implemented and compared with previous protocols. The simulation results indicate that SCAMA can support a much larger user population with diverse traffic requirements. This paper is organized as follows. In Section 2, we describe in detail the physical layer of our SCAMA protocol. In Section 3, we describe the multiple access operations and characteristics of the proposed SCAMA protocol. The source models are described in Section 4. Experimental results and their interpretations are included in Section 5. The last section concludes the paper.

\section{The Channel-Adaptive Physical Layer}

\subsection{Wireless Channel Model}

The wireless communication environment considered in this paper is the reverse-link situation of a wireless system where a number of mobile terminals contend to transmit ATM cells to an ATM server. The wireless link between a mobile ATM terminal and the ATM server is characterized by two components, namely the microscopic fading component and the macroscopic shadowing component. Microscopic fading is caused by the superposition of multipath components and is therefore fluctuating in a fast manner (on the order of a few msec). Macroscopic shadowing is caused by terrain configuration or obstacles and is fluctuating in a relatively slow manner (on the order of one to two seconds).

Let $c(t)$ be the combined channel fading which is given by: $c(t)=c_{l}(t) c_{s}(t)$, where $c_{l}(t)$ and $c_{s}(t)$ are the long-term macroscopic and short-term microscopic fading components, respectively. Both $c_{s}(t)$ and $c_{l}(t)$ are random processes with coherent time on the order of a few milli-seconds and seconds, respectively.

\subsection{Variable Throughput Channel Coding}

Redundancy is incorporated to the information packet for error protection. To exploit the time-varying nature of the wireless channel, a variable rate channeladaptive physical layer is employed as illustrated in Fig. 1. Channel state information (CSI), $c(t)$, which is estimated ${ }^{\dagger}$ at the receiver, is fed back to the transmitter via a low-capacity feedback channel. Based on the CSI, the level of redundancy and the modulation constellation applied to the information cells are adjusted accordingly by choosing a suitable transmission mode ${ }^{\ddagger}$. Thus, the instantaneous throughput is varied according to the instantaneous channel state. In our study, a 6-mode variable rate adaptive bit-interleaved trellis coded modulation scheme (ABICM) is employed [7]. Transmission

\footnotetext{
+ In this paper, we assumed CSI is estimated by the pilot-symbol approach [7].

$\ddagger$ Transmission mode refers to the combination of channel encoding rate and modulation constellation level
} 


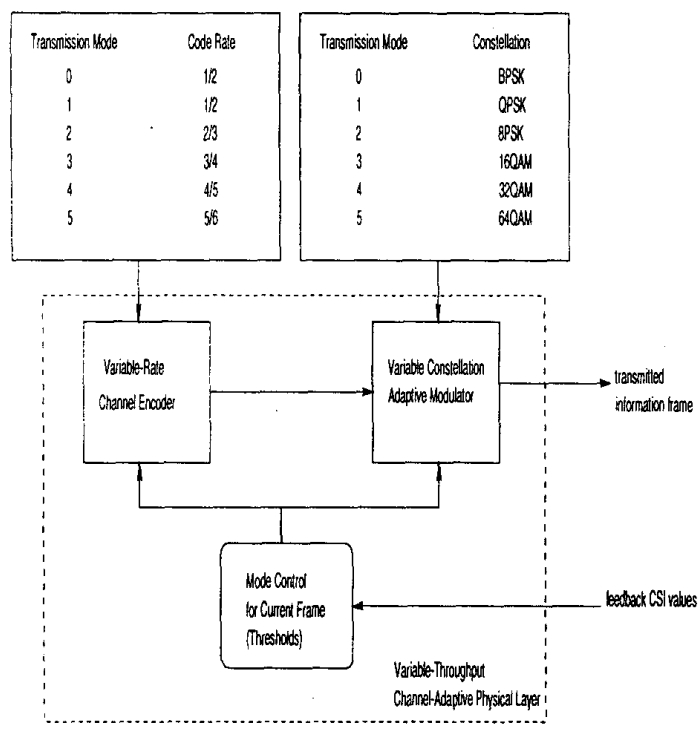

Fig. 1 A conceptual block diagram of the variable throughput channel adaptive physical layer.

modes with normalized throughput ${ }^{\dagger}$ varying from 0.5 to 5 are available depending on the channel condition. For real-time sources such as CBR or VBR, the physical layer employs a variable throughput forward error correction (FEC) code. For a non-real-time source such as $\mathrm{ABR}$, the physical layer employs a variable throughput error correction code embedded with error detection and retransmission.

Information cells per user are transmitted in the assigned traffic slots of a TDMA frame. Since the coherence time of short-term fading is around $20 \mathrm{msec}$, the CSI remains approximately constant within a traffic slot duration. Hence, all the transmitted symbols of the traffic slot (per user) share the same transmission mode, which is determined by the current CSI level. Specifically, transmission mode $q$ is chosen if the feedback CSI, $\hat{c}$, falls within the adaptation thresholds, $\left(\zeta_{q-1}, \zeta_{q}\right)$. Here, the operation and the performance of the ABICM scheme is determined by the set of adaptation thresholds $\left\{\zeta_{1}, \zeta_{2}, \ldots, \zeta_{n}\right\}$. Furthermore, we operate the ABICM scheme in the constant BER mode [7], [8]. That is, the adaptation thresholds are set optimally to maintain a target transmission error level over a range of CSI values. When the channel condition is good, a higher mode could be used and the system enjoys a higher throughput. On the other hand, when the channel condition is bad, a lower mode is used to maintain the target error level at the expense of a lower transmission throughput. Note that when the channel state is very

+ Normalized throughput refers to the number of information bits carried per modulation symbol. bad, the adaptation range of the ABICM scheme can be exceeded, making it impossible to maintain the targeted BER level.

\section{The SCAMA Protocol}

In the following, we describe our proposed fully channel-adaptive MAC protocol based on D-TDMA framing. The proposed protocol also provides a convenient structure to accommodate different service requirements of $C B R, V B R$, and $A B R$ effectively.

\subsection{Frame Structure}

Fig. 2 shows the uplink and the downlink frame structure of the proposed MAC protocol. To incorporate the channel-adaptive feature of the proposed MAC protocol, the TDMA frames for the uplink and downlink are divided into subframes as follows.

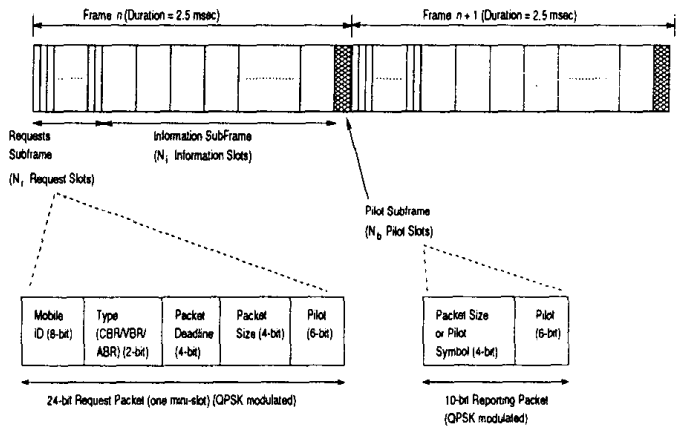

(a) uplink frame structure

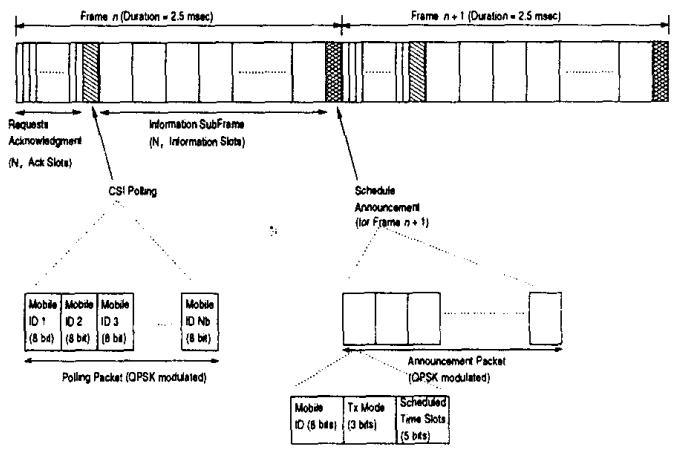

(b) downlink frame structure

Fig. 2 Frame structures of the proposed SCAMA protocol for CBR, VBR, and ABR users.

In the uplink, a frame is divided into three subframes. They are the request subframe, the traffic subframe, and the reporting subframe. Specifically, there are $N_{r}$ mini-slots in the request subframe for $\mathrm{CBR}, \mathrm{VBR}$, and $\mathrm{ABR}$ requests requests contention. Note that an $A B R$ user is not allowed to make reservation in the sense that even if an ABR user is granted traffic slot(s) in the current frame, it has to 
contend again in the next frame for the remaining data cells. On the other hand, CBR and VBR users can reserve slots in succeeding frames. Specifically, when a CBR or VBR user successfully makes a transmission request in one of the $N_{r}$ mini-slots, the user does not need to contend again in the next period and the request will be automatically generated in the MAC layer until the current burst ends. There are $N_{i}$ information slots in the traffic subframe for the transmission of $\mathrm{CBR}$, VBR, or ABR packets. Finally, there are $N_{b}$ mini-slots in the reporting subframe. The frame duration is $2.5 \mathrm{msec}$. Such a short frame duration has the advantage of shorter delay and is practicable in wideband systems [12].

A downlink FDD frame is similarly partitioned into four subframes, namely the acknowledgment subframe, the polling subframe, the traffic subframe, and the announcement subframe. The frame duration is also $2.5 \mathrm{msec}$ and the number of slots in the subframes are given by $N_{r}, N_{b}, N_{i}$, and $N_{b}$, respectively. Note that in both uplink and downlink, variable throughput adaptive channel coding and modulation is applied to traffic slots only. For the mini-slots of the other subframes, traditional QPSK modulation is applied.

\subsection{Protocol Operations}

The operation of the SCAMA protocol is divided into two phases, namely the request phase and transmission phase. In the request phase, mobile terminals which have packets to transmit will send a request packet in one of the $N_{r}$ request mini-slots, governed by the respective permission probability. The request packet is very short ( 24 bits), occupying only a mini-slot, as illustrated in Fig. 2(a). It contains the mobile terminal ID, request type (CBR, VBR, or $A B R$ ), data deadline, number of information data cells desired to transmit as well as pilot symbols for CSI estimation. If more than one mobile terminals send request packets in the same request mini-slot, collision occurs and all the request packets are lost if capture effect is not considered (if capture is considered, the request with the highest signal energy may be successfully received). After each request mini-slot, an acknowledgment packet will be broadcast from the base-station through the acknowledgment mini-slot in the downlink frame (see Fig. 3). The acknowledgment packet contains only the successful request packet ID. Mobile terminals that fail to receive an acknowledgment will retransmit the request packet in the next request mini-slot, again governed by the permission probability. On the other hand, successfully acknowledged users will wait for announcement on the allocation schedule of the traffic slots from the base-station.

Unlike traditional MAC protocols, the base-station

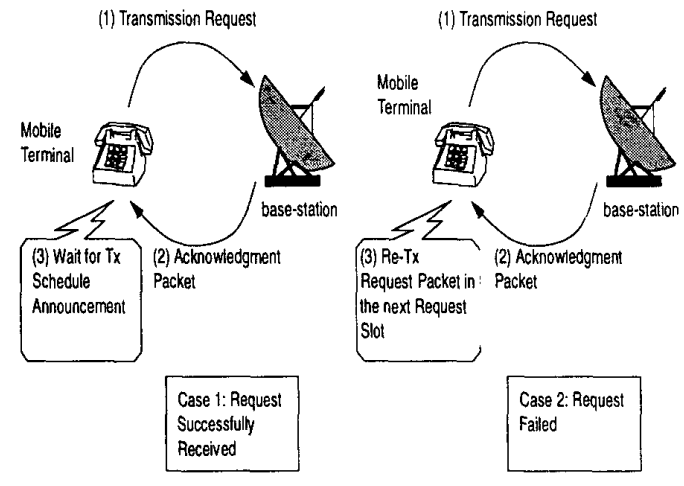

Fig. 3 Illustration of request and acknowledgment.

will collect all requests in the current request phase as well as the backlog requests from the previous frames before allocation of traffic slots. All the requests will be assigned priorities according to the deadline, CSI, service type (CBR, VBR, or ABR), as well as the waiting time of the request (i.e., the number of elapsed frames since the request is acknowledged), as shown in Fig. 4. Since the physical layer offers a variable throughput which is dependent on the CSI, the rationale behind the SCAMA MAC protocol is to give higher priority to the mobile terminals that are in better channel condition in the bandwidth allocation process. The motivation of this strategy is that a user with better channel condition, with the support of the variable rate channel encoder, can enjoy a larger throughput and therefore, can use the system bandwidth more effectively. Nevertheless, for fairness's sake, information slots should also be allocated to mobile terminals that are approaching their deadlines, despite their possibly worse channel states; otherwise, the queued information packets will be dropped.

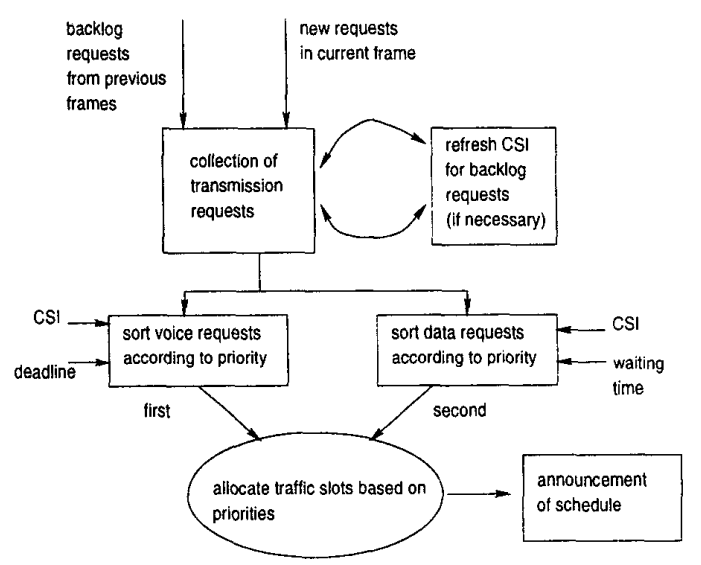

Fig. 4 Time-slot allocation procedure. 


\subsection{Priority Function for Slots Allocation\}}

In the SCAMA protocol, we employ a general priority function which could provide a flexible balance of the above conflicting goals. Furthermore, the slots allocation mechanism is also very flexible for incorporating other types of allocation algorithms such as variants of weighted fair queueing.

Specifically, the priority metric of the $i$-th request (which may be a new request or a backlog request), $\mu_{i}$, is given by the following equations:

$$
\mu_{i}=\left\{\begin{array}{l}
f_{\rho}\left(C S I^{i}\right)+\lambda_{C B R}\left(T_{d}^{i}\right)^{-\beta_{C B R}}+\Delta_{C B R} \\
f_{\rho}\left(C S I^{i}\right)+\lambda_{V B R}\left(T_{d}^{i}\right)^{-\beta_{V B R}}+\Delta_{V B R} \\
f_{\rho}\left(C S I^{i}\right)+\lambda_{A B R}\left(T_{w}^{i}\right)^{\beta_{A B R}}
\end{array}\right.
$$

where $T_{d}^{i}, T_{w}^{i}, \lambda_{C B R}, \lambda_{V B R}, \lambda_{A B R}, \beta_{C B R}, \beta_{V B R}, \beta_{A B R}$, $\Delta_{C B R}$, and $\Delta_{V B R}$ are the deadline, the waiting time, the forgetting factors of the $C B R, V B R$ and $A B R$ requests, as well as the priority offsets assigned to the CBR, VBR, or ABR users, respectively. The first term is aimed to enforce that a higher priority for requests with a higher throughput. The second term is to maintain fairness $^{\dagger}$ within each of the service classes. Finally, the last term is responsible for maintaining priority between different classes.

Thus, in the allocation phase, traffic slots are allocated to service requests according to the sorted priority metrics. If there are not sufficient traffic slots to service all requests, remaining requests are queued and re-considered in the next frame ${ }^{\ddagger}$. After the request phase, the results of traffic slot allocation will be broadcast in the announcement subframe of the downlink frame. The announcement packet contains the traffic slot allocation schedule as well as the transmission mode as illustrated in Fig. 2(b). Mobile terminals will then start to transmit information packets on the allocated traffic slot(s).

\subsection{Handling Heterogeneous Users Requirements}

The SCAMA protocol is reservation based for CBR and VBR users only. As mentioned earlier, for a $A B R$ user, even if traffic slots have been assigned for its successfully acknowledged request, the allocation is meant only for the current frame and the $A B R$ user has to initiate another request for any remaining data cells. By contrast, for a CBR or VBR user, when traffic slots have been assigned for its successfully

\footnotetext{
+ The second term will be large for requests with a urgent deadline or long waiting time.

$\ddagger$ If the deadline for a remaining request has expired, this request will not be queued anymore. The information packet at the mobile terminal will be dropped.
}

acknowledged request, additional requests will be automatically generated by the base-station (hence, reservation) periodically at $20 \mathrm{msec}$ (i.e.. taking voice as an example CBR source) and $40 \mathrm{msec}$ (i.e., taking video as an example VBR source) time intervals for CBR and VBR respectively. Thus, the CBR or VBR user does not need to contend for request mini-slots anymore in the current talkspurt. By avoiding unnecessary requests, the advantage of this reservation strategy is the reduction of the contention collisions in the request phase. For a CBR user, the number of cells generated per CBR period is constant and hence, this basic reservation scheme works fine. However, for a VBR user, the number of cells generated per VBR period is a random variable and hence, this information needs to be updated per VBR period in order to make proper reservation for VBR. The mechanism for VBR updating is illustrated in the following section.

\subsection{CSI Determination}

On the other hand, a critical component in the proposed SCAMA MAC protocol is the determination of the current CSI for each user. As mentioned earlier, we assume that the coherence time for short-term fading is around $20 \mathrm{msec}$ as illustrated in Section 2 while the frame duration is only $2.5 \mathrm{msec}$. Thus, the CSI remains approximately constant for at least two frames. For a new request, known pilot symbols are embedded in the request packets so that the current CSI can be estimated at the base station and this estimated CSI is valid for the next few frame duration. However, for a backlog request, the estimated CSI value obtained previously during a past request phase may be obsolete and thus, a mechanism is needed to obtain update the CSI

At the beginning of each frame, the base-station short-lists $N_{b}$ backlog requests (those with the CSI values expired or those requiring VBR reservation update) according to their priorities. A polling packet is then broadcast to the mobile terminals in the polling subframe. The CSI polling packet contains the mobile terminal IDs that are short-listed by the base-station. The structure of the polling packet is shown in Fig. 2(b). Mobile terminals listed in the polling packet respond at the appropriate reporting mini-slot according to the order specified in the polling packet. If the short-listed request is of CBR type with CSI value expired, the mobile station will transmit pilot symbols in the reporting mini-slot. Otherwise, the mobile station will transmit a VBR demand update packet as shown in Fig. 2(a). The VBR demand update packet contains the number of VBR cells generated in the current frame as well as pilot symbols for CSI updating.

Thus, as shown in Fig. 5, the base station could update the VBR reservation requests as well as backlog requests' CSI values (which are valid for at 
least two consecutive frames). The estimated CSI value is used to determine the transmission mode in the physical layer as well as to determine the priority of the request in the MAC layer. With the above considerations, the SCAMA protocol is outlined below in pseudo-code format.

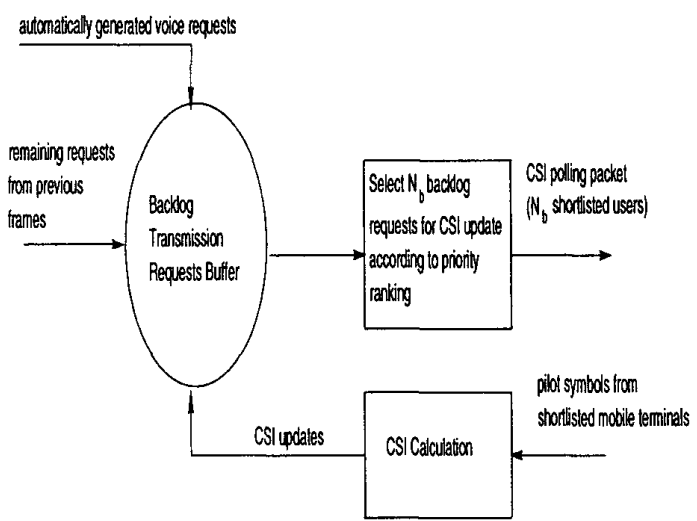

Fig. 5 CSI refreshing procedure.

\section{Source Models}

\subsection{CBR Source Model}

We use voice as an example CBR source. The voice source is assumed to be continuously toggling between the talkspurt and silence states. The duration of a talkspurt and a silence period are assumed to be exponentially distributed with means $t_{t}$ and $t_{s}$ seconds, respectively (as indicated by the empirical study in [9], $t_{t}=1$, and $t_{s}=1.35$ ). We assume a talkspurt and a silence period start only at a frame boundary. Finally, as mentioned above, a voice source cannot tolerate a cell loss rate higher than $1 \%$ in order to achieve a reasonable service quality [3]

\subsection{VBR Source Model}

We use video teleconference as an example VBR source. In the model we use [6], the number of cells per VBR period (i.e., $40 \mathrm{msec}$ for a $25 \mathrm{fps}$ frame rate) is govern by the DAR(1) model, which is a Markov chain characterized by three parameters: the mean, the variance, and $\rho$. The transition matrix is computed as: $P=\rho I+(1-\rho) Q$, where $\rho$ is the autocorrelation coefficient and $I$ is the identity matrix. Furthermore, each row of $Q$ is identical and consists of the negative binomial probabilities $\left(f_{o}, \ldots, f_{K}, F_{K}\right)$, where $F_{K}=\sum_{k<K} f_{k}$, and $K$ is the peak rate. Similar to a voice source, a video source can only tolerate a $1 \%$ cell loss rate [6].

\subsection{ABR Source Model}

The arrival time of data generated by a ABR data terminal is assumed to be exponentially distributed with mean equal to one second. The data size, in terms of number of cells, is also assumed to be exponentially distributed with mean equal to 100 cells. An ABR user will not drop cells because there is no deadline constraint. Again we assume that the cells arrive at a frame boundary.

\subsection{Terminal Contention Model}

As in most previous studies, to avoid excessive collisions, even if a user has some cells awaiting to be sent, the user will attempt to send a request at a request mini-slot only with a certain permission probability. The permission probability for CBR, VBR and ABR users are denoted by $p_{c}, p_{v}$, and $p_{u}$, respectively. The protocol also has a request queue which stores the previous requests that survive the contention but are not allocated information slots.

\section{Results and Interpretations}

In this section, we present the performance results of our proposed protocol. In our simulation study, we assume a transmission bandwidth of $1.36 \mathrm{MHz}$ for the TDMA frames. CBR bit rate is $8 \mathrm{kbps}$ while the average VBR bit rate is $128 \mathrm{kbps}$. We assume $N_{r}=10, N_{i}=40$, and $N_{b}=20$. Furthermore, we take $p_{c}, p_{v}$, and $p_{a}$, to be $0.3,0.3$, and 0.2 , respectively. Finally, the number of simulated frames is $2 \times 10^{6}$. Capture effect [2] is ignored.

We compare the MAC performance of the proposed SCAMA protocol with well-known ATM MAC protocols such as DQRUMA [4] and DTDMA/ PR [9]. However, one may argue that the performance gain of the proposed scheme is entirely due to the variable-throughput physical layer, which has been shown to offer a larger average throughput compared with traditional fixed-throughput physical layers [1] To better illustrate the superiority of our scheme, we compare with an additional MAC protocol, namely the DTDMA/VR [5], which employs the same variablethroughput physical layer as ours. All the three protocols incorporate a piggybacking mechanism for VBR users to update their traffic demands. All of the schemes are compared based on the same bandwidth, BER level and the average transmitted power.

\subsection{CBR Performance}

The service quality for CBR users, being voice sources, is governed by the average cell loss rate which is contributed by two factors: cell dropping at the mobile and cell loss during transmission. On one hand voice cell is delay sensitive and hence, voice cells are labeled with deadlines. A voice cell has to be discarded if its delay exceeds the deadline ${ }^{\dagger}$. Such discarding

$\dagger$ In this paper, the deadline of voice cell is assumed to be 20 msec after it is generated by the source. 
constitutes the cell dropping at the voice terminal. On the other hand, transmitted cells could be corrupted due to channel error and thus, cell transmission error results.

Fig. 6 shows the cell loss rate performance of our proposed SCAMA protocol as well as the other three previous protocols. In this set of experiments, we fixed the number of $A B R$ users to be 25 and that of VBR users to be 25 also. As can be seen, the DQRUMA and DTDMA/VR protocols perform much worse compared with the DTDMA/PR and SCAMA protocols. Indeed, the former two protocols achieve a cell loss rate which is higher than the $1 \%$ tolerance threshold for a voice source, while the latter two offer a two order of magnitude lower cell loss rate. This can be explicated by the fact that the DQRUMA and DTDMA/VR protocols do not incorporate any prioritization mechanism in the time slots allocation process. That is, the time slots are essentially allocated in a first-come-first-serve manner. Thus, as VBR and $A B R$ users usually have a much larger chunk of cells for transmission, the CBR users suffer from the "convoy" effect (i.e., a large request blocks a number of subsequent small requests), and thus, cell droppings occur quite frequently. The latter two protocols, however, allocate slots according to the requests' priorities. In particular, isochronous users such as CBR and VBR users are given a higher priority than the ABR users. The cell loss due to dropping can be avoided almost completely.

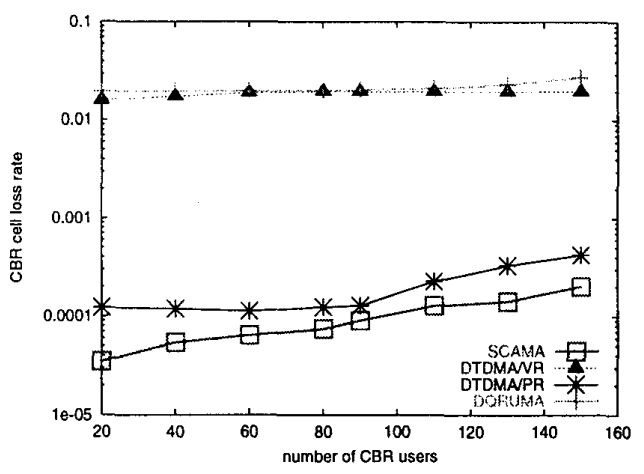

Fig. 6 Performance of CBR users.

\subsection{VBR Performance}

Similar to CBR users, the service quality of VBR users is also sensitive to loss (e.g., the image quality degrades if some cells are lost in a vidoe-conferencing application). Also, being a isochronous source, cells have deadlines such that missing the transmission deadlines render the cells useless. Thus, we also evaluate the performance of the protocols using the cell loss rate for VBR users. Fig. 7 depicts the VBR cell loss rate performance of the four protocols. We can see that in contrast to CBR performance, the DTDMA/PR protocol performs quite poorly for VBR users in that the cell loss rate is much higher than the $1 \%$ threshold even for 5 VBR users. This phenomenon is a result of assigning priority to CBR users in the DTDMA/PR, which concurs with the results presented in [9]. The DQRUMA protocol also gives similar performance as the DTDMA/PR protocol. The DTDMA/VR protocol, however, outperforms the previous two protocols by a large margin for VBR users. This is because the variable-throughput physical layer offers almost two times the average throuhgput compared with traditional fixed-throughput physical layers and is reflected in the performance of DTDMA/ VR. Finally, we can see that the proposed SCAMA protocol considerably outperforms the DTDMA/VR protocol. This illustrates the synergy that could be achieved by the judicious requests prioritization (based on CSI, urgency, and throughput) process in the former.

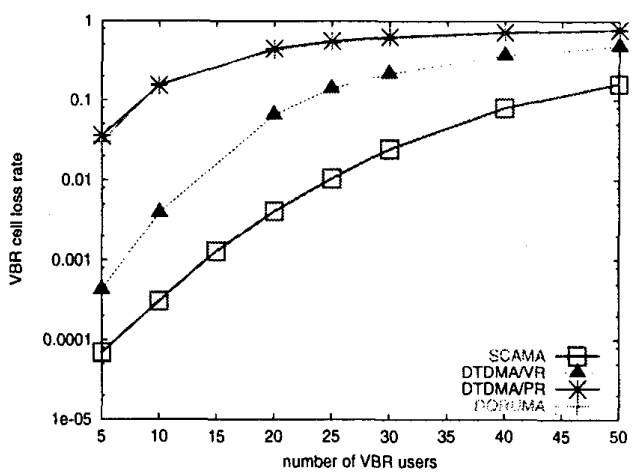

Fig. 7 Performance of VBR users.

\subsection{ABR Performance}

$A B R$ cells are delay insensitive and as such, they will not be discarded at the mobiles. However, ABR cells may experience transmission errors when the channel condition is poor. Thus, lost cells are retransmitted (through the data-link layer). This inevitably introduces additional delay due to retransmissions. Here, different from CBR and VBR users, ABR users' performance is quantified by two measures: delay and throughput. The average ABR data throughput is defined as the average number of cells successfully received at the base-station per frame. The average delay is defined as the average time that a cell spends waiting in the transmitter buffer until the beginning of the successful transmission. Fig. 8 illustrates the performance of data terminal in terms of delay and throughput, respectively. When the traffic load is high, the system is in a highly congested state so that the average per-user throughput drops and the average per-user delay also increases dramatically. These adverse phenomena are detrimental to the data 
users' quality of service (QoS), which depends critically on the parameters pair (delay, throughput) as describe above. Before the system gets into the congested state, the proposed SCAMA protocol consistently offers a much lower delay and a much higher throughput compared to the other three protocols. In other words, given a certain QoS level, the SCAMA protocol can support a much larger ABR user population. For example, at a QoS level of (1 sec, 0.25 ), the SCAMA protocol can support more than $200 \mathrm{ABR}$ users while the second best protocol, the DTDMA/VR protocol, can only support 100 ABR users.

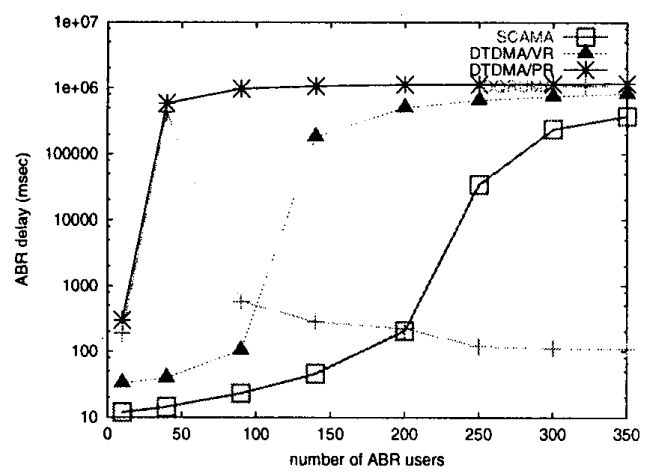

(a) ABR delay

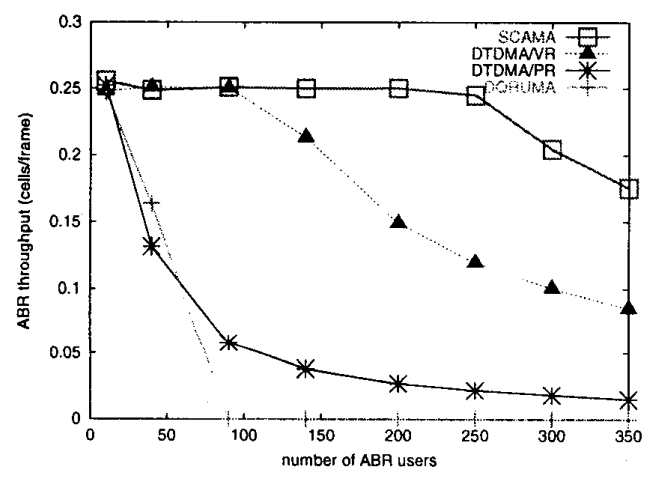

(b) ABR throughput

Fig. 8 Performance of ABR users.

\subsection{Interpretations}

From the simulation results shown above, the proposed SCAMA protocol is robust and outperforms two recently proposed efficient protocols by a considerable margin. In this section, we further provide some interpretations of the performance results.

First, a CBR terminal may experience a deep fading for a long time when it is affected by shadowing. In the other three protocols (including DTDMA/VR), bandwidth allocation in the MAC layer is carried out regardless of the current channel condition as detected in the physical layer. Thus, information slots could also be allocated to such a user and the transmitted packets will be very likely lost due to the poor channel condition. In other words, assigned slots are simply wasted. This kind of wasteful allocation is avoided in the SCAMA protocol.

Secondly, selection diversity is implicitly incorporated in the SCAMA protocol. Through the priority-based assignment process, every frame is packed with a selected group of information cells with good channel states. Thus, the effective delivered throughput per frame achieved in SCAMA can be much higher than that in DTDMA/VR and other protocols. In SCAMA, a large number of transmission requests are collected first before allocation of information slots. From the collection of requests, there is a high likelihood that a sufficient number of requests with good channel states can be selected to fully utilize the information slots in an effective manner (i.e., high throughput). For those requests with poor instantaneous channel states, their transmissions are deferred until when the CSI improves or the deadlines are approaching. By contrast, in the DTDMA/VR and DQRUMA protocols, requests are served in a first-come-first-serve manner due to the traditional strategy of immediately assigning slots upon successful receipt of requests. Thus, the channel states of such requests are highly diverse and, most importantly, some requests with bad channel states (hence very low throughput) are also served, whereby causing inefficient the bandwidth utilization. For example, a CBR terminal may experience a very good CSI for a long time (out of shadowing). In protocols without considering CSI in the prioritization process (DTDMA/PR) or those even without explicit prioritization (DTDMA/VR and DQRUMA), this user, however, may fail to successfully transmitted a request to the base-station, probably because of excessive collisions in the request phase. In comparison, our proposed scheme gathers a large number of requests through successive frames, and allocate time slots to the users that can use the system bandwidth more effectively. Thus, the likelihood of "missing" a user with good channel state is much lower and the utilization of bandwidth is therefore higher.

\section{Conclusions}

We have presented a new channel-adaptive MAC protocol for wireless ATM systems. The proposed protocol, called SCAMA, employs a judicious prioritization and scheduling mechanism, which takes into account all the critical performance parameters such as channel condition, urgency, and throughput. In particular, the prioritization is robust and flexible in that it can be easily adapted to suit the needs of a certain class of users (e.g., the CBR users). Our simulation results indicate that the performance of the 
SCAMA protocol is superior to three state-of-the-art wireless ATM MAC protocols.

\section{Acknowledgments}

The authors would like to thank Professor Victor Li for his initial inspiration on the idea of joint design of protocol layers. This research was also jointly supported by research initiation grants from the HKU CRCG under contract numbers 10202523 and 10202518 , and by a grant from the Hong Kong Research Grants Council under contract number HKU7124/99E.

\section{References}

[1] S. M. Alamouti and S. Kallel, "Adaptive TrellisCoded Multiple-Phase-Shift Keying for Rayleigh Fading Channels," IEEE Transactions on Communications, vol. 42, no. 6, pp. 2305-2314, June 1994.

[2] J. C. Ambak and W. van Blitterswijk, "Capacity of Slotted ALOHA in Raleigh Fading Channels," IEEE Journal on Selected Areas in Communications, vol. SAC-5, no. 2, pp. 261-268, Feb. 1987.

[3] J. Gruber and L. Strawczynski, "Subjective Effects of Variable Delay and Speech Clipping in Dynamically Managed Voice Systems," IEEE Transactions on Communications, vol. COM-33, no. 8, pp. 801-808, Aug. 1985.

[4] M. J. Karol, Z. Liu, and K. Y. Eng, "DistributedQueueing Requests Update Multiple Access (DQRUMA) for Wireless Packet (ATM) Networks," Proceedings of INFOCOM'95, pp. 1224-1231, 1995.

[5] M. Kawagishi, S. Sampei, and N. Morinaga, "A Novel Reservation TDMA Based Multiple Access Scheme Using Adaptive Modulation for Multimedia Wireless Communication Systems," Proceedings of VTC'98, pp. 112-116, 1998.

[6] T. V. Lakshman, A. Ortega, and A. R. Reibman, "VBR Video: Tradeoffs and Potentials," Proceedings of the IEEE, vol. 86, no. 5, pp. 952973, May 1998.

[7] V. K. N. Lau, "Performance of Variable Rate BitInterleaved Coding for High Bandwidth Efficiency," Proceedings of VTC'2000, Tokyo, May 2000, accepted for publication and to appear.

[8] V. K. N. Lau and Y.-K. Kwok, "Joint Design of Adaptive Channel Coding and Cell Scheduling for Wireless ATM," IEE Electronics Letters, vol. 36, no. 6, pp. 549-551, Mar. 2000.

[9] X. Qiu, V. O. K. Li, and J.-H. Ju, "A Multiple Access Scheme for Multimedia Traffic in Wireless ATM," ACM/Baltzer Mobile Networks and Applications, vol. 1, pp. 259-272, 1996.

[10]D. Raychaudhuri, "Wireless ATM Networks: Technology Status and Future Directions,"
Proceedings of the IEEE, vol. 87, no. 10, pp. 1790-1806, October 1999.

[11] J. Sanchez, R. Martinez, and M. W. Marcellin, "A Survey of MAC Protocols Proposed for Wireless ATM," IEEE Network, vol. 11, no. 6, pp. 52-62, Nov./Dec. 1997.

[12]A. Urie, M. Streeton, and C. Mourot, "An Advanced TDMA Mobile Access System for UMTS," Proceedings of the IEEE PIMRC'94, 1994. 